\title{
Fertilidade do solo e nutrição de tangerineiras 'Ponkan' manejados com resíduos sólidos e adubação química
}

\author{
Carlos H. Santos $^{1}$, Hélio G rassi Filho ${ }^{2}$, Jacqueline C. Santos ${ }^{2} \&$ Bruna B. Penteado $^{2}$
}

\section{RESUMO}

O objetivo do trabal ho foi avaliar alguns atributos químicos do solo e o estado nutricional das tangerineiras 'Ponkan' no solo manejado com resíduos orgânicos e adubação química. 0 experimento foi conduzido no Departamento de Recursos Naturais/Ciência do Solo - FCA/U N ESP em Botucatu, SP. As mudas de tangerineira foram plantadas e manejadas com lodo de esgoto (LE), esterco de curral curtido (EC) e adubação química (AQ). O s resultados permitiram concluir que houve um aumento significativo nos teores dos atributos CTC, MO, N-total P e K, no solo manejado com resíduo orgânico. A aplicação do lodo de esgoto também não prejudicou a qualidade do solo pois a adição dos elementos $\mathrm{As}, \mathrm{Cd}, \mathrm{Cr}$ e $\mathrm{Hg}$ em sua constituição, não foi significativa. $O$ manejo do solo com os resíduos proporcionou um aumento significativo no teor de N, P, Ca e S nas folhas das tangerineiras 'Ponkan' e, ao mesmo tempo, esses resíduos não contribuíram para um aumento significativo do teor foliar dos micronutrientes $\mathrm{B}, \mathrm{Cu}, \mathrm{Fe}$, $\mathrm{Mn}$ e $\mathrm{Zn}$ nem dos elementos $\mathrm{As}, \mathrm{Cd}, \mathrm{Cr}$ e $\mathrm{Pb}$. O manejo do solo com lodo de esgoto não resultou em aumento significativo dos elementos $\mathrm{As}, \mathrm{Cd}, \mathrm{Cr}$ e $\mathrm{Hg}$ nas folhas e nos frutos.

Palavras-chave: nutrição mineral, matéria orgânica, lodo de esgoto, mineralização

\section{Soil fertility and nutritional status of 'Ponkan' mandarin under solid organic residues and chemical fertilization}

\begin{abstract}
The objective of this research was to evaluate some soil chemical attributes and the nutritional status of 'Ponkan' mandarin managed with organic residues and chemical fertilization. The experiment was carried out at the Natural Resource D epartment - Soil Science Area, in the College of Agronomic Sciences - UNESP/Botucatu, SP. 'Ponkan' Mandarin seedlings were cropped and managed using the organic residues sewage sludge, cattle manure and chemical fertilization. The results allow the conclusion that there was an increase in the CEC, O M, N-total, $\mathrm{P}$ and $\mathrm{K}$ values in the soil managed with organic residues. The sewage sludge application did not harm the soil quality because the input of elements $\mathrm{As}, \mathrm{Cd}, \mathrm{Cr}$ and $\mathrm{Hg}$ present in its constitution were not significant. The soil management with organic residues increased significantly contents of $\mathrm{N}, \mathrm{P}, \mathrm{Ca}$ and $\mathrm{S}$ in 'Ponkan' leaves but did not contribute to increase significantly the elements $\mathrm{B}, \mathrm{Cu}, \mathrm{Fe}, \mathrm{Mn}, \mathrm{Zn}, \mathrm{As}, \mathrm{Cd}, \mathrm{Cr}$ and $\mathrm{Pb}$. The soil management with sewage sludge did not result in significant increase in the $\mathrm{As}, \mathrm{Cd}, \mathrm{Cr}$ and $\mathrm{Hg}$ content of leaves and fruits.
\end{abstract}

Key words: mineral nutrition, organic matter, sewage sludge, mineralization

\footnotetext{
${ }^{1}$ D outor, Faculdade de Ciências Agrárias/U N OESTE, Rodovia Raposo Tavares, km 572, Campus II, CEP 19067-175, Presidente Prudente, SP. Fone: (18) 3229-2000, ramal 2077. E-mail: chenrique@unoeste.br

2 FCA/U N ESP, Rua José Barbosa de Barros 1780, Fazenda Experimental Lageado, CEP 18610-307, Botucatu, SP. Fone: (14) 3811-7169. E-mail: heliograssi@fca.unesp.br; jac_camilo@yahoo.com.br; bpenteado@brenntagla.com
} 


\section{INTRODUÇÃO}

A elevação do custo dos fertilizantes comerciais e o aumento da poluição ambiental fazem do uso de resíduos orgânicos na agricultura uma opção atrativa, do ponto de vista econômico, em razão da ciclagem de nutrientes. Esses fatos geram um aumento na demanda por informações com o intuito de avaliar a viabilidade técnica e econômica para a disposição de alguns desses resíduos em solos agrícolas. Além disso, existem muitos resíduos orgânicos de origem urbana, industrial e agrícola, que podem ser usados na agricultura (Melo et al., 2008).

O lodo de esgoto, por exemplo, é um resíduo produzido a partir do tratamento, higienização, estabilização e secagem do esgoto urbano, com o objetivo de torná-lo menos poluído e permitir o seu retorno ao ambiente (Melo et al., 2001).

No caso de solos agrícolas, o uso de resíduos, em virtude de conterem altos teores de matéria orgânica, contribui para o maior armazenamento de C, aumento da CTC, maior complexação de elementos tóxicos, melhoria da estrutura, maior infiltração e retenção de água no solo, entre outros (Rocha et al., 2004) constituindo-se, assim, em componentes fundamentais para o aumento da capacidade produtiva do solo.

Desta forma, existem motivos importantes para viabilizar do ponto de vista social, ambiental e econômico a utilização deste resíduo (Silva et al., 2002). Porém o conhecimento de sua constituição química se torna imperativo (Melo et al., 2008).

O lodo de esgoto urbano tem sido utilizado na melhoria da qualidade de solos em áreas de florestas (Guedes et al., 2006), na recuperação de áreas degradadas (Modesto et al., 2009), como fertilizante nas culturas da soja (Lemainski \& Silva, 2006), do trigo (Chueiri et al., 2007) do milho (Silva et al., 2002) da cana-de-açúcar (Chiaradia et al., 2009) além do algodoeiro (Pedroza et al., 2006).

Com base nas informações relatadas objetivou-se, neste trabalho, avaliar alguns atributos componentes da fertilidade do solo e o estado nutricional das tangerineiras 'Ponkan', em solo manejado com lodo de esgoto, esterco de curral e adubação química.

\section{Material e MÉTODOS}

O experimento foi conduzido no Departamento de Recursos Naturais - Área de Ciência do Solo, da Faculdade de Ciências Agronômicas - UNESP/Campus de Botucatu, SP. O clima da região é definido como $\mathrm{Cwb}$, de clima quente com inverno seco, verão chuvoso e brando, conforme classificação de Köppen (Tubelis \& Salibe, 1989).

As mudas de tangerineira 'Ponkan' (Citrus reticulata Blanco) com 18 meses de idade, enxertadas em citrumeleiro 'Swingle' (Citrus paradisi Mcf. x Poncirus trifoliata Raf.) foram plantadas na segunda quinzena de dezembro de 2001, em recipientes com capacidade para $500 \mathrm{dm}^{3}$ de solo, distribuídas na área seguindo o espaçamento de 4 x $4 \mathrm{~m}$. Utilizou-se, nesses recipientes, um solo identificado como Latossolo Vermelho, de acordo com a classificação da EMBRAPA (2006). A caracterização dos atributos químicos do solo (Tabela 1) foi executada de acordo com metodologia de Raij et al. (2001).

Após a coleta do solo e 45 dias antes do plantio realizou-se uma calagem da terra dos recipientes, a $20 \mathrm{~cm}$ de profundidade, utilizando-se calcário dolomítico, com o objetivo de elevar a saturação por bases a 70\%. Cada recipiente foi irrigado para possibilitar a reação do calcário, sendo a operação repetida a cada sete dias até o momento do plantio.

Os tratamentos se constituíram na aplicação de $10 \mathrm{dm}^{3} \mathrm{de}$ lodo de esgoto (LE), na cova de plantio e em quatro adubações de cobertura, $10 \mathrm{dm}^{3}$ de esterco de curral (EC) curtido na cova de plantio e em quatro adubações de cobertura e apenas adubação química (AQ). Os resíduos foram aplicados em base úmida (Tabela 2). Esses tratamentos foram implementados seguindo-se o delineamento estatístico em blocos casualizados, com três repetições e três plantas/repetição.

Na ocasião do plantio foi realizada uma adubação química do solo nas parcelas manejadas com os resíduos orgânicos com o objetivo de fornecimento de nutrientes para o desenvolvimento inicial das mudas de tangerina. Na parcela representante do tratamento com adubação química não foi adicionado nenhum tipo de resíduo orgânico.

O lodo de esgoto utilizado no plantio das tangerineiras foi coletado na estação de tratamento de esgoto da SABESP, localizada no município de Franca, SP, e o esterco de curral curtido foi originado na FCA/UNESP, em Botucatu, SP. A caracterização química dos resíduos orgânicos, conforme metodologia de análise do LANARV (1988), está apresentada na Tabela 2.

Decorridos trinta dias do plantio, juntamente com a adubação de cobertura química convencional, a qual seguiu a recomendação do GPACC (1996), também foram aplicados 10 $\mathrm{dm}^{3}$ de lodo de esgoto e $10 \mathrm{dm}^{3}$ de esterco de curral curtido, ambos em base úmida (Tabela 2), nas respectivas parcelas. Nesta ocasião, os resíduos apresentavam as mesmas características daqueles aplicados no plantio.

Posteriormente ao plantio e a cada seis meses, realizaram-se as adubações de cobertura com o lodo de esgoto, com o resíduo produzido na Opersan Serviços Ambientais SC Ltda, Jundiaí, SP, e com o esterco de curral curtido coletado na FCA/UNESP (ambos em base úmida). As análises químicas dos resíduos estão na Tabela 2.

No primeiro ano de manejo das plantas utilizou-se, em todos os tratamentos, a recomendação de adubação mineral de Malavolta \& Violante Netto (1988) e a do GPACC (1996) para a realização da adubação de cobertura, aplicando-se $80 \mathrm{~g}$ de $\mathrm{N}$ na forma de uréia e $20 \mathrm{~g}$ de $\mathrm{K}_{2} \mathrm{O}$ na forma de nitrato de potássio,

Tabela 1. Atributos químicos básicos do solo anterior à implantação do experimento

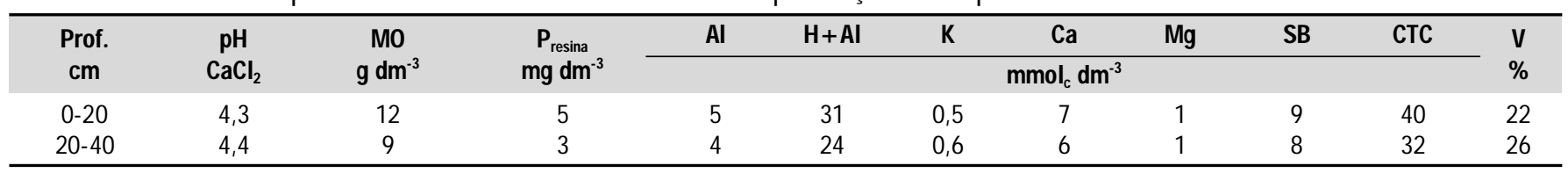


Tabela 2. Caracterização química dos resíduos orgânicos utilizados no manejo do solo*

\begin{tabular}{|c|c|c|c|c|c|c|c|c|c|c|}
\hline \multirow{2}{*}{ Parâmetros } & \multicolumn{5}{|c|}{ Lodo de esgoto* } & \multicolumn{5}{|c|}{ 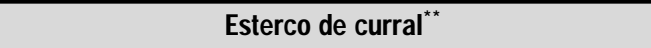 } \\
\hline & $\mathbf{P}$ & $\mathrm{C}_{1}$ & $C_{2}$ & $C_{3}$ & $\mathrm{C}_{4}$ & $\mathbf{P}$ & $\mathrm{C}_{1}$ & $\mathrm{C}_{2}$ & $C_{3}$ & $\mathrm{C}_{4}$ \\
\hline $\mathrm{pH}\left(\mathrm{CaCl}_{2}\right)$ & 7,5 & 7,5 & 5,0 & 6,5 & 3,6 & 4,3 & 4,3 & 4,1 & 4,2 & 8,5 \\
\hline Mat. Org. $\left(\mathrm{g} \mathrm{kg}^{-1}\right)$ & 367 & 367 & 550 & 530 & 550 & 320 & 320 & 280 & 230 & 460 \\
\hline Um. $110^{\circ} \mathrm{C}\left(\mathrm{g} \mathrm{kg}^{-1}\right)$ & 660 & 660 & 110 & 50 & 520 & 550 & 550 & 410 & 90 & 100 \\
\hline C-total $\left(\mathrm{g} \mathrm{kg}^{-1}\right)$ & 350 & 350 & 306 & 271 & 306 & 178 & 178 & 156 & 128 & 256 \\
\hline $\mathrm{N}$-total $\left(\mathrm{g} \mathrm{kg}^{-1}\right)$ & 50,0 & 50,0 & 42,0 & 34,0 & 28,0 & 10,5 & 10,5 & 14,0 & 11,2 & 12,2 \\
\hline $\mathrm{P}_{2} \mathrm{O}_{5}\left(\mathrm{~g} \mathrm{~kg}^{-1}\right)$ & 25 & 25 & 20,2 & 16,0 & 18,0 & 8,7 & 8,7 & 13,2 & 8,6 & 10,0 \\
\hline $\mathrm{K}_{2} \mathrm{O}\left(\mathrm{g} \mathrm{kg}^{-1}\right)$ & 1,5 & 1,5 & 1,4 & 1,6 & 2,20 & 2,1 & 2,1 & 2,6 & 0,7 & 6,0 \\
\hline $\mathrm{Ca}\left(\mathrm{g} \mathrm{kg}^{-1}\right)$ & 14,6 & 14,6 & 12,6 & 10,6 & 15,0 & 6,1 & 6,1 & 11,1 & 5,9 & 8,0 \\
\hline $\mathrm{Mg}\left(\mathrm{g} \mathrm{kg}^{-1}\right)$ & 2,0 & 2,0 & 1,2 & 1,7 & 2,5 & 0,8 & 0,8 & 1,8 & 1,12 & 5,0 \\
\hline $\mathrm{Cu}\left(\mathrm{mg} \mathrm{kg}^{-1}\right)$ & 200 & 200 & 148,2 & 192 & 662 & 154 & 154 & 89 & 140 & 102 \\
\hline $\mathrm{Mn}\left(\mathrm{mg} \mathrm{kg}^{-1}\right)$ & 158 & 158 & 654 & 464 & 646 & 348 & 348 & 236 & 318 & 538 \\
\hline $\mathrm{Fe}\left(\mathrm{mg} \mathrm{kg}^{-1}\right)$ & 2.250 & 2.250 & 3.540 & 1.850 & 3.050 & 2.010 & 2.010 & 1.820 & 2.467 & 1.750 \\
\hline $\mathrm{Zn}\left(\mathrm{mg} \mathrm{kg}^{-1}\right)$ & 676 & 676 & 789 & 1.020 & 1.700 & 216 & 216 & 329 & 268 & 560 \\
\hline As $\left(\mu \mathrm{gg}^{-1}\right)$ & 1.728 & 1.728 & 976 & 1.643 & 275 & 150 & 150 & 212 & 1.273 & 271 \\
\hline $\mathrm{Cd}(\mu \mathrm{g} \mathrm{kg})$ & 0,06 & 0,06 & 1,34 & nd & 2,22 & nd & nd & nd & nd & nd \\
\hline $\mathrm{Cr}\left(\mu \mathrm{g} \mathrm{kg}^{-1}\right)$ & 190 & 190 & 147 & 5,9 & nd & 14,6 & 14,6 & 6,2 & 128,2 & 5,0 \\
\hline $\mathrm{Hg}\left(\mu \mathrm{g} \mathrm{kg}^{-1}\right)$ & nd & $\mathrm{Nd}$ & 0,53 & 0,04 & 0,05 & 0,03 & 0,03 & nd & nd & 0,05 \\
\hline $\mathrm{Pb}\left(\mu \mathrm{g} \mathrm{kg}^{-1}\right)$ & 61 & 61 & 145 & 30,1 & nd & 2,7 & 2,7 & 3,5 & 5,1 & nd \\
\hline
\end{tabular}

* Resultados médios, de cinco repetições, com base na matéria seca

** P - Plantio (Dez/2001); C - 1 adubação de cobertura (J an/2002); C - 2a adubação de cobertura (J ul/2002); C - 3a adubação de cobertura (J an/2003); $C_{4}$ - 4a adubação de cobertura (J ul/2003). nd - valor não determinado em função do teor do elemento no resíduo e da sensibilidade do equipamento de leitura

parceladas em $15-20-30-35 \%$ do total de $\mathrm{N}$ e $20-30-50 \%$ da quantidade total de $\mathrm{K}_{2} \mathrm{O}$. As adubações foram parceladas em quatro fertirrigações $(15,20,30$ e $35 \%$ de N), aos 30, 60, 90 e 120 dias após o plantio (dap) e três (20, 30 e $50 \%$ de $\left.\mathrm{K}_{2} \mathrm{O}\right)$, aos 60, 90 e 120 dap. Nos segundo e terceiro anos de cultivo a adubação do solo também foi realizada via fertirrigação, seguindo-se a recomendação presente do GPACC (1996). Apesar de haver fornecimento de $\mathrm{N}$ através da mineralização dos materiais orgânicos optou-se, no segundo ano, por adicionar $\mathrm{N}$ na forma mineral nesses tratamentos; já no terceiro ano e em função da disponibilidade de $\mathrm{N}$, interrompeu-se o fornecimento de $\mathrm{N}$ mineral aos solos manejados com os resíduos orgânicos.

A irrigação para a manutenção do desenvolvimento das plantas foi proporcionada por um sistema de irrigação por gotejamento, sendo instalado um gotejador por planta, com vazão de $24 \mathrm{~L} \mathrm{~h}^{-1}$, a 1,8 Bar de pressão. As linhas dos gotejadores foram dispostas a uma distância de $25 \mathrm{~cm}$ do caule das plantas. Objetivou-se a manutenção do potencial da água no solo próximo à capacidade de campo.

Para avaliar os atributos químicos do solo foi concluída, em março de 2004, a última etapa de coleta das amostras de terra e se amostraram as profundidades de 0-20 e 21-40 cm, de todas as parcelas, na região do bulbo formado pelo gotejador, cuja referência foi a projeção da copa. Essas amostras foram homogeneizadas, secadas em estufa com circulação forçada de ar a $45^{\circ} \mathrm{C}$ e peneiradas em malha de $2 \mathrm{~mm}$ visando à obtenção de duas amostras compostas para cada repetição por tratamento e por profundidade. Nessas amostras se determinaram os parâmetros: Matéria Orgânica (MO) $\left(\mathrm{g} \mathrm{dm}^{-3}\right)$, $\mathrm{P}\left(\mathrm{mg} \mathrm{dm}^{-3}\right), \mathrm{K}, \mathrm{Ca}, \mathrm{Mg}$ e CTC $\left(\mathrm{mmol}_{\mathrm{c}} \mathrm{dm}^{-3}\right)$ segundo metodologia descrita por Raij et al. (2001) e o N total, de acordo com metodologia de Cantarella \& Trivelin (2001). Os micronutrientes $\mathrm{B}, \mathrm{Cu}, \mathrm{Fe}, \mathrm{Mn}$ e Zn também foram determinados segundo a metodologia de Raij et al. (2001). A determinação dos elementos $\mathrm{As}, \mathrm{Cd}, \mathrm{Cr}, \mathrm{Hg}$ e $\mathrm{Pb}$ foi realizada conforme resolução da norma P4.230 da CETESB (1999) e de acordo com metodologia descrita por Abreu \& Andrade (2001).

Realizou-se a avaliação do estado nutricional das plantas em março de 2004, através da coleta de vinte e sete amostras de folhas fisiologicamente maduras, retiradas de ramos frutíferos, de todas as plantas, na altura mediana da copa e em cada quadrante. Cumprida esta etapa realizou-se a análise dos elementos N, P, K, Ca, Mg, S, B, Cu, Fe, Mn, Zn, As, Cd, Cr, Hg e $\mathrm{Pb}$, segundo metodologia citada por Malavolta et al. (1997).

O teor dos referidos metais também foi analisado nos frutos produzidos no ano de 2004, para o que se escolheram quatro frutos/repetição/tratamento. Os frutos foram colhidos e processados para a obtenção das frações: casca, suco e bagaço. As frações casca e bagaço foram colocadas em estufa com circulação forçada de ar a $55^{\circ} \mathrm{C}$, durante $72 \mathrm{~h}$, para a secagem completa. Todas as frações foram utilizadas para a determinação dos elementos $\mathrm{As}, \mathrm{Cd}, \mathrm{Cr}, \mathrm{Hg}$ e $\mathrm{Pb}$, de acordo com metodologia descrita por Malavolta et al. (1997).

A quantificação dos elementos $\mathrm{As}, \mathrm{Cd}, \mathrm{Cr}, \mathrm{Hg}$ e $\mathrm{Pb}$ nos extratos obtidos do preparo das amostras de material vegetal e de terra foi realizada através do equipamento de absorção atômica com forno de grafite acoplado.

Os resultados analíticos foram submetidos à análise de variância pelo Teste F avaliando-se o efeito dos tratamentos e a comparação entre as médias foi realizada pelo teste de Tukey, a um nível máximo de $5 \%$ de probabilidade.

\section{RESULTADOS E DISCUSSÃO}

Houve um incremento significativo nos valores de matéria orgânica no solo manejado com lodo de esgoto, comparado com os demais tratamentos, de $0-20 \mathrm{~cm}$ de profundidade (Tabela 3 ). Resultados semelhantes foram encontrados por Simonete et al. (2003), Bovi et al. (2007), Chiba et al. (2008) e Trannin et al. (2008). Os valores expressos na Tabela 3 não refletem, porém, 
Tabela 3. Resultados médios de atributos químicos do solo em função da profundidade e do manejo do solo

\begin{tabular}{|c|c|c|c|c|c|c|c|c|c|c|c|c|c|c|}
\hline \multirow{4}{*}{ Trat. } & \multirow{2}{*}{\multicolumn{2}{|c|}{$\begin{array}{c}\text { CTC } \\
\mathrm{mmol}_{\mathrm{c}} \mathrm{dm}^{-3}\end{array}$}} & \multirow{2}{*}{\multicolumn{2}{|c|}{$\begin{array}{l}\text { M. } 0 . \\
\mathrm{g} \mathrm{dm}^{-3}\end{array}$}} & \multicolumn{2}{|c|}{ N-total } & \multicolumn{2}{|c|}{$\mathbf{P}$} & \multicolumn{2}{|c|}{$K$} & \multicolumn{2}{|c|}{$\mathrm{Ca}$} & \multicolumn{2}{|c|}{$\mathrm{Mg}$} \\
\hline & & & & & \multicolumn{4}{|c|}{$\mathrm{mg} \mathrm{dm^{-3 }}$} & \multicolumn{6}{|c|}{$\mathrm{mmol}_{\mathrm{c}} \mathrm{dm}^{-3}$} \\
\hline & $0-20$ & $20-40$ & $0-20$ & $20-40$ & $0-20$ & $20-40$ & $0-20$ & $20-40$ & $0-20$ & $20-40$ & $0-20$ & $20-40$ & $0-20$ & $20-40$ \\
\hline & \multicolumn{14}{|c|}{$\mathrm{cm}$} \\
\hline LE & 20,7 & 59,40 & $16,1 \mathrm{a}$ & $13,3 \mathrm{a}$ & $92,6 a$ & $61,6 a$ & $229,7 a$ & $142,1 \mathrm{a}$ & $3,3 a b$ & 3,8 & 5,4 & 3,1 & 1,3 & 0,3 \\
\hline EC & 16,5 & 54,30 & $15,4 \mathrm{a}$ & $11,3 \mathrm{~b}$ & $60,6 a$ & $49,2 \mathrm{~b}$ & $116,3 \mathrm{~b}$ & $159,3 \mathrm{a}$ & 4,3 a & 3,6 & 4,1 & 1,8 & 1,3 & 0,6 \\
\hline$A Q$ & 13,4 & 50,00 & $10,0 \mathrm{~b}$ & $10,0 \mathrm{~b}$ & $43,7 \mathrm{~b}$ & $44,1 \mathrm{~b}$ & $79,3 \mathrm{C}$ & $60,8 b$ & $3,0 \mathrm{~b}$ & 3,3 & 4,4 & 2,5 & 3,5 & 1,6 \\
\hline $\mathrm{CV}(\%)$ & 17,4 & 13,70 & 21,3 & 20,1 & 22,50 & 16,2 & 37,7 & 58,8 & 33,9 & 33,2 & 60,4 & 67,3 & 79,9 & 75,3 \\
\hline DMS & 12,5 & 10,07 & 2,84 & 2,50 & 14,80 & 8,37 & 90,3 & 95,39 & 8,7 & 1,5 & 8,2 & 3,6 & 6,7 & 3,13 \\
\hline
\end{tabular}

todo o potencial de incremento de matéria orgânica apresentado pela adição desses resíduos ao solo (Tabela 2).

Apesar de não se ter detectado a diferença estatística nos valores de CTC entre os tratamentos (Tabela 3), a influência da porção orgânica do lodo de esgoto mostrou estar associada à alteração do complexo coloidal destacando-se, segundo Melo et al. (2001), que em solos de regiões de clima tropical e subtropical seu emprego se torna viável para o manejo do solo, pelo fato de ser fonte de matéria orgânica, e ainda, poder melhorar a CTC. Bovi et al. (2007), Simonete et al. (2003) e Trannin et al. (2008) verificaram que a adição de lodo de esgoto em dose superior a 20 tha $^{-1}$ aumentou o teor de matéria orgânica e CTC efetiva do solo manejado; entretanto, Silva et al. (2001) destacaram que o aumento nos valores dos parâmetros citados pode ocorrer de forma mais pronunciada algum tempo após o início do manejo, além do que pode ser um efeito de curta duração, o que dependerá da quantidade e da qualidade dos resíduos adicionados ao solo.

Os solos manejados com resíduos orgânicos apresentaram maior teor de N-total (Tabela 3). O incremento de $\mathrm{N}$-total no solo a favor da aplicação do lodo de esgoto foi de aproximadamente $112 \%$ representando, portanto, uma reserva possível de ser mineralizada. O manejo com esterco de curral também proporcionou, embora com menor amplitude, um incremento significativo de $38,8 \%$ no teor de $\mathrm{N}$-total no solo comparado com a adubação química. Bovi et al. (2007) corroboram com esses resultados e ainda citaram que a mineralização do $\mathrm{N}$ orgânico é proporcional à dose aplicada no solo.

Acompanhando a distribuição da matéria orgânica associada com a adição de $\mathrm{N}$ ao longo das profundidades amostradas, nos solos manejados com resíduos orgânicos os teores de Ntotal, de 21-40 cm de profundidade (Tabela 3), são inferiores àqueles obtidos de $0-20 \mathrm{~cm}$, uma vez que a matéria orgânica adicionada às adubações de cobertura foi incorporada apenas superficialmente. Oliveira et al. (2001) também não verificaram incrementos significativos de $\mathrm{N}$-total em profundidades abaixo de $30 \mathrm{~cm}$, evidenciando pouca ou nenhuma movimentação desta forma de $\mathrm{N}$ no perfil do solo. Segundo Trannin et al. (2008) a aplicação de lodo de esgoto em grande quantidade proporcionou aumento quadrático do teor de $\mathrm{N}$ total nas camadas de 0-20 $\mathrm{cm}$ e linear a $21-40 \mathrm{~cm}$ de profundidade.

A diagnose foliar das tangerineiras (Tabela 4) mostrou que houve um aumento significativo nos valores de $\mathrm{N}$ nas folhas das plantas manejadas com lodo de esgoto e com esterco de curral, comparado com o manejo de adubação química, mostrando o potencial de fornecimento de $\mathrm{N}$ pela adição dos resíduos orgânicos (Tabela 2) ao solo.

Tabela 4. Resultados médios do teor de $\mathrm{N}, \mathrm{P}, \mathrm{K}, \mathrm{Ca}, \mathrm{Mg}$ e S $\left(\mathrm{g} \mathrm{kg}^{-1}\right)$ nas fol has de tangerineiras 'Ponkan' enxertadas em citrumeleiro 'Swingle', em função do manejo do solo

\begin{tabular}{|c|c|c|c|c|c|c|}
\hline \multirow{2}{*}{ Tratamento } & $\mathbf{N}$ & $\mathbf{P}$ & $\mathbf{K}$ & $\mathrm{Ca}$ & Mg & $\mathbf{S}$ \\
\hline & \multicolumn{6}{|c|}{$\mathrm{g} \mathrm{kg}^{-1}$} \\
\hline Lodo de es goto (LE) & $28,8 \mathrm{a}$ & $2,1 \mathrm{a}$ & $13,8 \mathrm{a}$ & $19,5 \mathrm{a}$ & $4,6 a$ & $3,3 a$ \\
\hline Esterco de curral (EC) & $27,5 \mathrm{a}$ & $2,0 a$ & $15,0 \mathrm{a}$ & $16,3 \mathrm{~b}$ & $4,5 \mathrm{a}$ & $2,4 \mathrm{~b}$ \\
\hline Adubação química (AQ) & $25,0 \mathrm{~b}$ & $1,7 \mathrm{~b}$ & $18,3 \mathrm{a}$ & $12,5 \mathrm{C}$ & $4,4 a$ & $2,3 \mathrm{C}$ \\
\hline CV $(\%)$ & 2,38 & 9,36 & 7,22 & 7,96 & 6,22 & 6,40 \\
\hline DMS & 1,27 & 0,22 & 2,53 & 2,53 & 0,60 & 0,25 \\
\hline
\end{tabular}

Médias seguidas de letras distintas nas colunas diferem entre si pelo teste de Tukey, ao nível de $5 \%$ de probabilidade

No tratamento representado pela adubação química os valores de $\mathrm{N}$ nas folhas se encontraram na faixa considerada adequada para o desenvolvimento das plantas cítricas (23-27 $\mathrm{g} \mathrm{kg}^{-1}$ ), de acordo com Quaggio et al. (1997); entretanto, existiu um teor adicional de $14 \mathrm{e} 12 \%$ de N, respectivamente, nas folhas das plantas cultivadas no solo manejado com lodo de esgoto e com esterco de curral. Segundo Mattos Júnior et al. (2004) a produção de fruto tangor 'Murcott', com qualidade desejável ao mercado, correlacionou-se positivamente com o teor foliar de $\mathrm{Nem}$ torno de $29,0 \mathrm{~g} \mathrm{~kg}^{-1}$, valores obtidos neste experimento, com o lodo de esgoto.

No Egito, a aplicação de $5 \mathrm{dm}^{3}$ de lodo por planta como fonte de $\mathrm{N}$, aumentou a produção de laranja, limão e tangerina, em média 60\% (Darwish et al., 1997). Na Espanha também se constatou que o emprego de lodo de esgoto em pomar de laranjeira 'Valência', nas doses de $12 \mathrm{Mg} \mathrm{ha}^{-1}$ ano $^{-1}$ durante sete anos consecutivos, proporcionou efeitos no solo e na planta semelhantes aos do manejo realizado apenas com fertilizantes minerais (Canet et al., 1997). De acordo com Bovi et al. (2007) a aplicação de lodo de esgoto no plantio refletiu em aumento do teor foliar de N. Almeida et al. (2005) corroboraram com todos esses resultados, salientando que as tangerineiras 'Ponkan' apresentaram melhor vigor vegetativo quando cultivadas em solo manejado com esterco de curral em cobertura. Para Panzenhagen et al. (1999) e Fidalski \& Stenzel (2006) o desenvolvimento das plantas e a produção de frutos cítricos de qualidade estão diretamente relacionados ao manejo do solo com matéria orgânica.

O manejo do solo realizado com lodo de esgoto e esterco de curral também proporcionou um aumento significativo de 103,7 e 27,4\%, respectivamente, no teor de $\mathrm{P}$, a $20 \mathrm{~cm}$ de profundidade, 
superando os resultados proporcionados pelo manejo com adubação química (Tabela 3). A diferença significativa ocorreu entre os manejos do solo com resíduo orgânico, destacando que a adição de $\mathrm{P}$ via adubação com lodo foi $59,9 \%$ superior ao resultado obtido com a aplicação de esterco de curral. Esta diferenciação no teor de $\mathrm{P}$ no solo entre os manejos está relacionada com o maior teor de matéria orgânica encontrado nos solos manejados com lodo $(122,9 \%)$ e esterco de curral $(77,1 \%)$ o que favoreceu a maior disponibilização do $\mathrm{P}$ às plantas. De acordo com Melo et al. (2001), o lodo de esgoto é uma fonte potencial de fornecimento de $\mathrm{P}$, sendo grande parte deste elemento proveniente de compostos polifosfatados, além da biomassa microbiana. O teor de Pé um dos atributos químicos de solos ácidos, influenciado positivamente pela aplicação de resíduos como o lodo de esgoto (Abreu Júnior et al., 2005). Simonete et al. (2003), Chiba et al. (2008) e Trannin et al. (2008) corroboraram com esses resultados.

A análise química das amostras de terra da profundidade de 21-40 cm (Tabela 3) mostrou que houve diminuição do teor de P comparado com os dados obtidos nas amostras coletadas na profundidade de $0-20 \mathrm{~cm}$. Apesar da diminuição nos valores de $\mathrm{P}$, não ocorreu diferença significativa entre os tratamentos com resíduo mas, sim, entre ambos e o manejo com adubação química. Destaca-se que os níveis de P no solo, apresentados na Tabela 3, são considerados muito elevados, de acordo com a classe de teores, para as culturas perenes (GPACC, 1996).

O teor de $\mathrm{P}$ nas folhas das tangerineiras 'Ponkan' também refletiu o efeito da adição do lodo de esgoto e do esterco de curral no solo. Notou-se uma diferença significativa entre os manejos com resíduos orgânicos e com adubação química, com um incremento de $23,8 \%$ de $\mathrm{P}$ nas folhas das plantas que foram tratadas com o lodo e $16,3 \%$ nas folhas das tangerineiras associadas ao esterco de curral (Tabela 3). Com exceção do manejo de adubação química, os demais são considerados acima da faixa ideal (1,2-1,6 $\mathrm{g} \mathrm{kg}^{-1}$, Quaggio et al., 1997) para o desenvolvimento das plantas cítricas.

A adição de lodo de esgoto poderia ter proporcionado um resultado melhor comparado com o esterco de curral; entretanto, segundo com Dynia et al. (2006) variações na concentração de nutrientes disponíveis nos solos estão relacionadas com a taxa de mineralização dos resíduos orgânicos.

No solo tratado com lodo na camada de $0-20 \mathrm{~cm}$, constatouse um teor de K 20,3 e 42,3\% inferior aos tratamentos com adubação química e com esterco, respectivamente, embora não se tenha detectado diferença significativa entre os mesmos (Tabela 3). A única diferença observada foi a relacionada aos tratamentos com esterco de curral e adubação química. $\mathrm{O}$ balanço de íons $\mathrm{Ca}$ e $\mathrm{Mg}$, introduzidos no sistema através da calagem e dos resíduos (Tabela 3), em relação ao K em solução, pode ter promovido maior ocupação dos sítios de troca nos colóides do solo, ocasionando maiores chances de lixiviação do K através da água de irrigação. De acordo com Silva et al. (1998) a aplicação de lodo de esgoto nas doses de 15 e $30 \mathrm{Mg}$ ha $^{-1}$, provocou decréscimos de 6,9 e 17,2\% nos teores de $\mathrm{K}$ e $\mathrm{Mg}$, respectivamente, comparados com os da adubação química sem, entretanto, alterar a classe de teores no solo, estabelecida para a cultura da cana-de-açúcar; na ocasião, os autores ainda citaram que no total de $\mathrm{K}$ aplicado via lodo de esgoto $\left(1,5 \mathrm{~g} \mathrm{~kg}^{-1} \mathrm{de}\right.$ $\mathrm{K})$ apenas $3 \%$ foram considerados trocáveis e, portanto, extraídos pela resina.

Quando se consideraram as amostras de $21-40 \mathrm{~cm}$ (Tabela 3), observou-se a ocorrência de um acúmulo maior do K, reforçando a hipótese da sua lixiviação em função da presença de altos teores de $\mathrm{Ca}$ e $\mathrm{Mg}$ e irrigação constante; a exceção foi constatada para os teores de K obtidos nas amostras do manejo apenas com adubação química.

Como se pôde observar, mesmo com a adição de $\mathrm{K}$ ao solo (Tabela 3), o lodo de esgoto não foi uma fonte adequada de fornecimento deste nutriente, concordando com Simonete et al. (2003) e Bovi et al. (2007). Em uma situação avaliada por Kitamura et al. (2008) o K foi significativamente diferente entre os manejos com e sem matéria orgânica, permanecendo estável em teores considerados baixos.

Os resultados (Tabela 4) mostraram que os teores de $\mathrm{K}$ nas folhas das tangerineiras cultivadas com resíduos orgânicos, estavam dentro da faixa considerada ideal para o seu desenvolvimento, ou seja, de 12-17 $\mathrm{g} \mathrm{kg}^{-1}$ (Malavolta \& Violante Netto, 1988).

Não se verificou diferença significativa entre os tratamentos com relação ao teor dos elementos $\mathrm{Ca}$ e $\mathrm{Mg}$ disponíveis no solo no período avaliado (Tabela 3). Silva et al. (2001) relataram que da incorporação ao solo de $175 \mathrm{Mg} \mathrm{ha}^{-1} \mathrm{de} \mathrm{Ca}$, através do lodo, apenas $6 \%$ deste total foram extraídos pela resina; entretanto, Trannin et al. (2008) observaram uma resposta positiva no aumento dos teores de $\mathrm{Ca}$ e $\mathrm{Mg}$ com a aplicação de logo de esgoto enquanto Chiba et al. (2008) não verificaram tais efeitos significativos, demonstrando controvérsias do manejo com relação a este assunto.

Na situação apresentada na Tabela 3, os teores de Ca no solo, a $20 \mathrm{~cm}$ de profundidade são, em todos os tratamentos, superiores ao estabelecido como normal para o desenvolvimento de plantas perenes, ou seja, acima de 7,0 $\mathrm{mmol}_{\mathrm{c}} \mathrm{dm}^{-3}$; os referidos valores também são oriundos dos procedimentos de calagem.

A diagnose foliar mostrou teor maior de $\mathrm{Ca}$ nas folhas das tangerineiras manejadas com lodo de esgoto (Tabela 4). Embora tenha ocorrido uma diferenciação significativa entre os tratamentos, seus teores se encontraram abaixo do nível considerado adequado para as plantas cítricas $\left(35,0-45,0 \mathrm{~g} \mathrm{~kg}^{-1}\right)$ (Quaggio et al., 1997), o que pode ser considerado normal neste estádio de intenso desenvolvimento, uma vez que não foram diagnosticados sintomas de deficiência nutricional.

Com relação ao $\mathrm{Mg}$, corroborando com esses resultados Rocha et al. (2004) citaram um aumento significativo do teor de Mg somente trinta e dois meses após a aplicação de altas doses do resíduo, na camada de $0-5 \mathrm{~cm}$. Com base nos resultados da Tabela 3 não foi possível observar diferença significativa no teor foliar, em função do manejo do solo. As tangerineiras cultivadas em todos os tratamentos, apresentaram um teor foliar de $\mathrm{Mg}$ próximo ao indicado como adequado (2,5-4,0 $\mathrm{g} \mathrm{kg}^{-1}$ ) (Quaggio et al., 1997). A Tabela 4 também mostrou um aumento significativo de $\mathrm{S}$ diante do manejo do solo com resíduos orgânicos.

O incremento significativo nos valores de matéria orgânica no solo tratado com lodo de esgoto, refletiu diretamente no 
Tabela 5. Resultados médios dos teores de micronutrientes $\mathrm{B}, \mathrm{Cu}, \mathrm{Fe}, \mathrm{Mn}$ e $\mathrm{Zn}\left(\mathrm{mg} \mathrm{dm}^{-3}\right)$ em função do manejo do solo e do cultivo com tangerineira 'Ponkan' enxertadas em citrumel eiro 'Swingle'

\begin{tabular}{|c|c|c|c|c|c|c|c|c|c|c|}
\hline \multirow{4}{*}{ Tratamento } & \multicolumn{2}{|c|}{ B } & \multicolumn{2}{|c|}{$\mathrm{Cu}$} & \multicolumn{2}{|c|}{$\mathrm{Fe}$} & \multicolumn{2}{|c|}{ Mn } & \multicolumn{2}{|c|}{$\mathrm{Zn}$} \\
\hline & \multicolumn{10}{|c|}{$\mathrm{mg} \mathrm{dm}^{-3}$} \\
\hline & $0-20$ & $21-40$ & $0-20$ & $21-40$ & $0-20$ & $21-40$ & $0-20$ & $21-40$ & $0-20$ & $21-40$ \\
\hline & \multicolumn{10}{|c|}{$\mathrm{cm}$} \\
\hline LE & $0,50 a$ & $0,38 \mathrm{a}$ & $7,46 \mathrm{a}$ & 3,85 & $189,9 a$ & $174,3 \mathrm{a}$ & $5,25 a$ & 1,23 & $22,01 a$ & 6,50 \\
\hline EC & $0,31 b$ & $0,30 \mathrm{~b}$ & $4,67 \mathrm{~b}$ & 4,85 & $111,4 \mathrm{~b}$ & $133,3 \mathrm{~b}$ & $2,26 b$ & 1,50 & $11,20 \mathrm{~b}$ & 6,60 \\
\hline$A Q$ & $0,25 \mathrm{c}$ & $0,21 \mathrm{c}$ & $3,18 \mathrm{c}$ & 4,38 & $61,8 \mathrm{C}$ & $91,3 \mathrm{c}$ & $1,13 \mathrm{C}$ & 0,96 & $7,50 \mathrm{~b}$ & 5,95 \\
\hline CV (\%) & 12,0 & 9,30 & 22,22 & 29,86 & 22,63 & 27,55 & 47,65 & 62,84 & 74,13 & 46,78 \\
\hline DMS & 0,04 & 0,03 & 1,14 & 1,30 & 32,20 & 36,69 & 1,38 & 0,77 & 10,08 & 2,97 \\
\hline
\end{tabular}

Médias seguidas de letras distintas nas colunas diferem entre si pelo teste de Tukey, ao nível de 5\% de probabilidade. LE - lodo de esgoto; EC - esterco de curral; AQ - adubação química

teor de micronutrientes (Tabela 5), a $20 \mathrm{~cm}$ de profundidade referindo-se, especialmente, aos elementos $\mathrm{B}, \mathrm{Cu}, \mathrm{Fe}, \mathrm{Mn}$ e zn. O reflexo positivo no teor desses elementos no solo está relacionado à presença dos mesmos em sua constituição, cujos dados corroboram com os encontrados por Simonete \& Kiehl (2002), Chiba et al. (2008) e Trannin et al. (2008).

A diagnose foliar não evidenciou efeito significativo nos teores foliares de B, Cu e Fe (Tabela 6) em função dos manejos. Destaca-se, porém, que os teores de $\mathrm{Cu}, \mathrm{Fe}, \mathrm{Mn}$ e $\mathrm{Zn}$ estavam mais elevados comparados com os critérios estabelecidos por Quaggio et al. (1997) mas, ao mesmo tempo, não se constataram sintomas de toxidez.

Tabela 6. Resultados médios dos teores dos micronutrientes $\mathrm{B}, \mathrm{Cu}, \mathrm{Fe}, \mathrm{Mn}$ e $\mathrm{Zn}\left(\mathrm{mg} \mathrm{kg}^{-1}\right)$ nas fol has de tangerineiras 'Ponkan' em função do manejo do solo

\begin{tabular}{lccccc}
\hline \multirow{2}{*}{ Tratamento } & B & Cu & Fe & Mn & Zn \\
\cline { 2 - 6 } & \multicolumn{5}{c}{ mg kg $^{-1}$} \\
Lodo de es goto (LE) & 86,5 & 36,75 & 278,0 & 120,50 a & 126,3 \\
Esterco de curral (EC) & 70,8 & 40,75 & 286,5 & 116,00 a & 118,0 \\
Adubação química (AQ) & 73,0 & 43,25 & 286,8 & 89,50 b & 106,8 \\
CV (\%) & 11,44 & 22,16 & 10,84 & 10,82 & 13,78 \\
DMS & 17,34 & 17,63 & 60,77 & 23,22 & 31,86 \\
\hline
\end{tabular}

Médias seguidas de letras distintas nas colunas diferem entre si pelo teste de Tukey, ao nível de $5 \%$ de probabilidade

A ausência de uma resposta significativa nos teores foliares de micronutrientes pela aplicação de resíduos orgânicos no solo (Tabela 6) pode ser atribuída aos fatores, como forte complexação que os elementos podem sofrer pela ação da matéria orgânica (Kabata-Pendias \& Pendias, 2001) e até mesmo na solução do solo, além de um efeito de diluição da concentração dos elementos na fitomassa. Neste contexto é importante citar que as plantas manejadas com lodo de esgoto e esterco de curral apresentaram um crescimento vegetativo maior na fase final do período de avaliação, podendo ter ocasionado uma diluição dos micronutrientes no tecido foliar, gerando valores próximos aos encontrados nas folhas das plantas adubadas apenas com adubação química. Informações semelhantes obtiveram Simonete \& Kiehl (2002) e Rangel et al. (2006).

O manejo do solo com os resíduos orgânicos não refletiu em efeito significativo nos teores disponíveis dos elementos As, $\mathrm{Cd}, \mathrm{Cr}, \mathrm{Hg}$ e $\mathrm{Pb}$, nas profundidades de 0-20 e 21-40 cm (Tabela 7). No caso do $\mathrm{Pb}$, não foi possível a determinação do teor disponível em nenhuma das profundidades amostradas, haja vista que, possivelmente, os teores do solo estavam abaixo do nível de detecção do método analítico utilizado indicando, portanto, que o teor disponível em cada tratamento foi muito baixo; já para os metais pesados $\mathrm{Cd}$ e $\mathrm{Cr}$, a mesma situação ocorreu na profundidade de $21-40 \mathrm{~cm}$.

Chiba et al. (2008) não observaram efeito significativo dos tratamentos nos teores totais dos metais pesados $\mathrm{Cd}, \mathrm{Cr}, \mathrm{Ni}$ e $\mathrm{Pb}$ no solo. Segundo os autores, a diferença entre os manejos parece estar relacionada à composição química do lodo de esgoto utilizado. Trannin et al. (2008) também verificaram que a aplicação do lodo de esgoto não exerceu efeito sobre os teores dos metais $\mathrm{Cd} \mathrm{e} \mathrm{Pb}$, tendo em vista que este resíduo continha baixos teores desses elementos e, mesmo com a aplicação da dose máxima de lodo, os teores desses metais se mantiveram muito abaixo dos limites estabelecidos pela CETESB (2005), em todas as profundidades de amostragem.

Para os solos do Estado de São Paulo, a CETESB (2005) determinou valores orientadores de referência para o monitoramento do acúmulo dos elementos como sendo: As: 3,50 mg kg-1, Cd: 0,5 mg kg-1 , Cr: $40 \mathrm{mg} \mathrm{kg}^{-1}, \mathrm{Hg}: 0,05 \mathrm{mg} \mathrm{kg}^{-1} \mathrm{e}$ $\mathrm{Pb}: 17 \mathrm{mg} \mathrm{kg}^{-1}$.

$\mathrm{O}$ fato do solo dos tratamentos com lodo e esterco apresentar teores de $\mathrm{As}, \mathrm{Cd}, \mathrm{Cr}, \mathrm{Hg}$ e $\mathrm{Pb}$ abaixo do limite de referência estabelecido pela CETESB (2005), pode estar relacionado com a própria quantidade disponível dos elementos

Tabela 7. Resultados médios dos teores de metais pesados $\mathrm{As}, \mathrm{Cd}, \mathrm{Cr}$ e Hg $\left(\mathrm{mg} \mathrm{dm}^{-3}\right)$ nas amostras de terra, em função do manejo do solo

\begin{tabular}{|c|c|c|c|c|c|c|c|c|}
\hline \multirow{4}{*}{ Tratamento } & \multicolumn{2}{|c|}{ As } & \multicolumn{2}{|c|}{$\mathrm{Cd}$} & \multicolumn{2}{|c|}{$\mathrm{Cr}$} & \multicolumn{2}{|c|}{$\mathrm{Hg}$} \\
\hline & \multicolumn{8}{|c|}{$\mathrm{mg} \mathrm{dm}^{-3}$} \\
\hline & $0-20$ & $20-40$ & $0-20$ & $20-40$ & $0-20$ & $20-40$ & $0-20$ & $20-40$ \\
\hline & \multicolumn{8}{|c|}{$\mathrm{cm}$} \\
\hline Lodo de esgoto (LE) & 0,22 & 0,01 & 0,01 & 0,00 & 0,08 & 0,00 & 0,08 & 0,01 \\
\hline Esterco de curral (EC) & 0,24 & 0,02 & 0,01 & 0,00 & 0,07 & 0,00 & 0,01 & 0,01 \\
\hline Adubação química (AQ) & 0,23 & 0,00 & 0,00 & 0,00 & 0,07 & 0,00 & 0,02 & 0,01 \\
\hline
\end{tabular}


existentes em cada resíduo (Tabela 2), com a complexação de um elemento em questão, pela própria matéria orgânica, pela adsorção a óxidos hidratados e pela quantidade, qualidade e estágio de decomposição da matéria orgânica aplicada.

Em relação ao aspecto da presença dos metais pesados no tecido vegetal, os resultados mostraram que não houve diferença significativa no teor dos metais pesados nas folhas das tangerineiras, em função dos tratamentos (Tabela 8).

Tabela 8. Resultados médios dos teores de metais pesados As, Cd, $\mathrm{Cr}$ e $\mathrm{Hg}\left(\mathrm{mg} \mathrm{kg}^{-1}\right)$ nas folhas de tangerineiras 'Ponkan' enxertadas em citrumeleiro 'Swingle', em função do manejo do solo

\begin{tabular}{lcccc}
\hline \multirow{2}{*}{ Tratamento } & As & Cd & $\mathbf{C r}$ & $\mathbf{H g}$ \\
\cline { 2 - 5 } & \multicolumn{4}{c}{$\mathbf{~ m g ~ k g ~}^{-1}$} \\
Lodo de esgoto (LE) & 1,55 & 0,08 & 0,05 & 0,03 \\
Esterco de curral (EC) & 1,84 & 0,06 & 0,06 & 0,01 \\
Adubação química (AQ) & 2,20 & 0,10 & 0,03 & 0,02 \\
CV (\%) & 41,8 & 53,2 & 55,3 & 48,5 \\
DMS & 0,93 & 0,06 & 0,04 & 0,03 \\
\hline
\end{tabular}

Médias seguidas de letras distintas, nas colunas, diferem entre si pelo teste de Tukey, ao nível de $5 \%$ de probabilidade

De todos os elementos analisados o As apresentou teores maiores nas folhas coletadas em todos os tratamentos (Tabela 8). De acordo com Melo et al. (1994) os níveis considerados tóxicos estão entre $15-50 \mathrm{mg} \mathrm{kg}^{-1}$, o que não refletiu a realidade dos dados deste experimento, enquanto os demais elementos $\mathrm{Cd}, \mathrm{Cr}$ e $\mathrm{Hg}$ foram encontrados em baixos teores nas folhas. Com relação ao Cd, Simonete et al. (2003) não o encontraram em folhas de Eucalyptus grandis nem em plantas de milho ou, quando os encontraram, os mesmos estavam abaixo do limite de detecção da metodologia analítica indicando que, naquelas condições, a fitodisponibilidade do Cd foi ínfima. Nogueira et al. (2008) salientaram que o Cd não foi detectado em todas as partes de milho estudadas, indicando, assim, baixo risco de contaminação pelo lodo de esgoto utilizado. Os valores considerados tóxicos para as plantas, de acordo com Melo et al. (1994) estão na faixa entre 2,0-8,0 $\mathrm{mg} \mathrm{kg}^{-1}$.

$\mathrm{Na}$ abordagem do Cr, Nogueira et al. (2008) também que não encontraram teor deste metal pesado em folhas, grãos nem na palha de milho cultivado em um solo que recebeu até $127 \mathrm{Mg}$ $\mathrm{ha}^{-1}$ de lodo de esgoto. Os valores estipulados como fitotóxicos, de acordo com Melo et al. (1994) estão em torno de $10 \mathrm{mg} \mathrm{kg}^{-1}$. $\mathrm{Na}$ mesma linha de pesquisa Chiba et al. (2008) relataram que os metais $\mathrm{Cd}, \mathrm{Cr}$ e $\mathrm{Pb}$ não foram detectados na parte aérea das plantas de cana-de-açúcar, provavelmente por se encontrarem em teores inferiores ao limite de detecção do método analítico $\left(0,01 \mathrm{mg} \mathrm{L}^{-1}\right)$.

A análise química das tangerinas também evidenciou baixos teores de metais pesados (Tabela 9), e denotou que não houve diferença significativa no teor de alguns elementos analisados nos frutos.

Torna-se imperativo destacar que, no procedimento analítico realizado, não foi possível determinar o teor dos elementos As, $\mathrm{Cd}$ e $\mathrm{Hg}$, independente da fração do fruto analisada (casca, bagaço ou suco), pois não atingiram o nível de detecção do método analítico, o qual se encontrava na faixa de $0,20 \mu \mathrm{g} \mathrm{kg}^{-1}$ para o $\mathrm{Cd}$ e $0,25 \mu \mathrm{g} \mathrm{kg}^{-1}$ para o As e $\mathrm{Hg}$. Da mesma forma, não
Tabela 9. Resultados médios dos teores de metais pesados $\mathrm{Cr}$ e $\mathrm{Pb}\left(\mu \mathrm{g} \mathrm{kg}{ }^{-1}\right)$ nas frações casca, bagaço e suco de tangerinas 'Ponkan' enxertadas em citrumeleiro 'Swingle', em função do manejo do solo

\begin{tabular}{lrrrrrr}
\hline \multirow{2}{*}{ Tratamento } & \multicolumn{5}{c}{$\mathbf{C r}$} & \multicolumn{3}{c}{$\mathbf{P b}$} \\
\cline { 2 - 7 } & \multicolumn{7}{c}{$\mathbf{C} \mathbf{~ k g}^{-1}$} \\
Lodo de esgoto (LE) & 145,0 & - & - & 210,0 & - & - \\
Esterco de curral (EC) & 213,0 & - & - & 205,0 & - & - \\
Adubação química (AQ) & 175,0 & - & - & 242,0 & - & - \\
CV (\%) & 61,4 & - & - & 58,9 & - & - \\
DMS & 73,2 & - & - & 42,9 & - & - \\
\hline
\end{tabular}

Médias seguidas de letras distintas diferem entre si pelo teste de Tukey, ao nível de $5 \%$ de probabilidade. Obs.: C - casca; B - bagaço; S - suco. * Não atingiram o nível de detecção do método analítico nas frações

foi possível a determinação dos metais $\mathrm{Cr}$ e $\mathrm{Pb}$ no suco e no bagaço, visto que não atingiram a sensibilidade do método, que foi de 0,25 e $0,50 \mu \mathrm{g} \mathrm{kg}^{-1}$ para os elementos $\mathrm{Cr}$ e $\mathrm{Pb}$, respectivamente; observou-se, porém, que dos elementos determinados a casca dos frutos apresentou maior teor de $\mathrm{Cr}$ e $\mathrm{Pb}$ (Tabela 9). Nogueira et al. (2008) também não detectaram o $\mathrm{Pb}$ nos grãos nem no sabugo de milho mas o mesmo elemento foi detectado nas demais partes da planta, principalmente nas doses de $90 \mathrm{Mg} \mathrm{ha}^{-1}$, porém não foram observados valores de $\mathrm{Pb}$ fitotóxicos.

Os resultados expressos nas Tabelas 8 e 9, além daqueles relatados na literatura, explicitam que a toxicidade exercida por metais pesados em plantas tem sido demonstrada somente quando os elementos são incorporados aos solos em formas solúveis ou quando os resíduos orgânicos contaminados são incorporados em taxas muito elevadas, além de se considerar o tempo de manejo.

\section{CONCLUSÕES}

1. Deu-se um aumento significativo nos teores dos atributos $\mathrm{MO}, \mathrm{N}$-total, $\mathrm{P}$ e $\mathrm{K}$ e $\mathrm{S}$ no solo manejado com resíduos orgânicos comparado àquele com adubação química.

2. A aplicação do lodo de esgoto não prejudicou a qualidade do solo pois a adição antrópica dos elementos $\mathrm{As}, \mathrm{Cd}, \mathrm{Cr}$ e $\mathrm{Hg}$, presentes em sua constituição, não foi significativa.

3. O manejo do solo com os resíduos proporcionou um aumento significativo no teor de N, P e Ca nas folhas das tangerineiras 'Ponkan' e, ao mesmo tempo, esses resíduos não contribuíram para um aumento significativo do teor foliar dos micronutrientes $\mathrm{B}, \mathrm{Cu}, \mathrm{Fe}, \mathrm{Mn}$ e $\mathrm{Zn}$, nem dos elementos $\mathrm{As}$, $\mathrm{Cd}, \mathrm{Cr}$ e $\mathrm{Pb}$.

4. O manejo do solo com lodo de esgoto não resultou em aumento significativo dos elementos $\mathrm{As}, \mathrm{Cd}, \mathrm{Cr}$ e $\mathrm{Hg}$ nas folhas e nos frutos das tangerineiras 'Ponkan'.

\section{Agradecimentos}

À Fundação de Amparo à Pesquisa do Estado de São Paulo - FAPESP, pelo auxílio financeiro. 


\section{LITERATURA CITADA}

Abreu, C. A. de; Andrade, J. C. de. Determinação de cobre, ferro, manganês, zinco, cádmio, níquel e chumbo em solos usando a solução de DTPA em pH 7,3. In: Raij, B. van; Andrade, J. C.; Cantarella, H.; Quaggio, J. A. (ed.). Análise química para avaliação da fertilidade de solos tropicais. 1.ed. Campinas: Instituto Agronômico, 2001. p.240-250.

Abreu Júnior, C. H.; Oliveira, F. C.; Silva, F. C.; Berton, R. S. Uso de resíduos orgânicos no pomar. Tópicos em Ciência do Solo, v.4, p.391-470, 2005.

Almeida, T. R. P. de; Leonel, S.; Tecchio, M. A.; Mischan, M. M. Formação do pomar de tangerineira 'Poncã', em função da adubação química e orgânica. Revista Brasileira de Fruticultura, v.27, n.2, p.288-291, 2005.

Bovi, M. L. A.; Goday Júnior, G.; Costa, E. A. D. da; Berton, R. S.; Spiering, S. H.; Vega, F. V. A.; Cembranelli, M. A. R.; Maldonado, C. A. B. Lodo de esgoto e produção de palmito em pupunheira. Revista Brasileira de Ciência do Solo, v.31, n.1, p.153-166, 2007.

Canet, R.; Pomares, F.; Tarazona, F. Chemical extractability and availability of heavy metals after seven years application of organic wastes to a citrus soil. Soil Use and Management, v.13,p.117-121, 1997.

Cantarella, H.; Trivelin, P. C. O. Determinação de nitrogênio inorgânico em solo pelo método da destilação a vapor. In: Raij, B. van; Andrade, J. C.; Cantarella, H.; Quaggio, J. A. (ed.). Análise química para avaliação da fertilidade de solos tropicais. 1.ed. Campinas: Instituto Agronômico, 2001. p.271276.

CETESB - Companhia de Tecnologia de Saneamento Ambiental. Aplicação de biossólido em áreas agrícolas: Critérios para projeto e operação São Paulo, 1999. 35p. Manual Técnico.

CETESB - Companhia de Tecnologia de Saneamento Ambiental. Dispõe sobre a aprovação dos valores orientadores para solos e águas subterrâneas no estado de São Paulo - 2005, em substituição aos valores orientadores de 2001. http:// ww w. cet e s b.s p.gov.br/solo/relatorios/ tabela_valores_2005.pdf. 25 jan. 2009.

Chiaradia, J. J.; Chiba, M. K; Andrade, C. A. de; Oliveira, C. de; Lavorenti; A. Produtividade e nutrição de mamona cultivada em área de reforma de canavial tratada com lodo de esgoto. Revista Brasileira de Ciência do Solo, v.33, n.3, p.701-709, 2009.

Chiba, M. K.; Mattiazzo, M. E.; Oliveira, F. C. Cultivo de canade-açúcar em argissolo tratado com lodo de esgoto. II fertilidade do solo e nutrição da planta. Revista Brasileira de Ciência do Solo, v.32, n.2, p.653-662, 2008.

Chueiri, W. A.; Serrat, B. M.; Biele, J.; Favaretto, N. Lodo de esgoto e fertilizante mineral sobre parâmetros do solo e de plantas de trigo. Revista Brasileira de Engenharia Agrícola e Ambiental, v.11, n.5, p.502-508, 2007.

Darwish, O. H.; Ahmed, F. F.; Mohamed, G. A. Fate of heavy elements in sludge when applied to fruit trees. Agriculture Research Journal, v.42, p.159-169, 1997.

Dynia, J. F.; Souza, M. D.; Boeira, R. C. Lixiviação de nitrato em Latossolo cultivado com milho após aplicações sucessivas de lodo de esgoto. Pesquisa Agropecuária Brasileira, v.41, n.5, p.855-862, 2006.
EMBRAPA - Empresa Brasileira de Pesquisa Agropecuária. Sistema Brasileiro de Classificação de Solos. 2.ed. Rio de Janeiro: EMBRAPA, 2006. 306p.

Fidalski, J.; Stenzel, N. M. C. Nutrição e produção da laranjeira "Folha Murcha" em porta-enxertos e plantas de cobertura permanente na entrelinha. Ciência Rural, v.36, n.3, p.807$813,2006$.

GPACC - Grupo Paulista de Adubação e Calagem para Citros. Recomendação de adubação e calagem para o estado de São Paulo. 2.ed. Campinas: Instituto Agronômico: 1996. 283p.

Guedes, M. C.; Andrade, C. A. de; Poggiani, F.; Mattiazzo, M. E. Propriedades químicas do solo e nutrição do eucalipto em função da aplicação de lodo de esgoto. Revista Brasileira de Ciência do Solo, v.30, n.2, p.267-280, 2006.

Kabata-Pendias, A.; Pendias, H. Trace elements in soil and plants. Boca Raton: CRC Press Inc., 1985. 315p.

Kitamura, A. E.; Alves, M. C.; Suzuki, L. G. A. S.; Gonzalez, A. P. Recuperação de um solo degradado com a aplicação de adubos verdes e lodo de esgoto. Revista Brasileira de Ciência do Solo, v.32, n.1, p.405-416, 2008.

LANARV - Laboratório Nacional de Referência Vegetal. Análise de corretivos, fertilizantes e inoculantes. Métodos oficiais. Brasília: Ministério da Agricultura, Secretaria Nacional de Defesa Agropecuária, 1988. 104p.

Lemainski, J.; Silva, J. E. da. Avaliação agronômica e econômica da aplicação de biossólido na produção de soja. Pesquisa Agropecuária Brasileira, v.41, n.10, p.1477-1484, 2006.

Malavolta, E.; Violante Netto, A. Nutrição mineral, calagem, gessagem e adubação dos citros. In: Simpósio de Citricultura, 1988, Jaboticabal. Anais...Jaboticabal: FUNEP, 1988. p.23384.

Malavolta, E.; Vitti, G. C.; Oliveira, S. A. Avaliação do estado nutricional de plantas: Princípios e aplicações. 2.ed. Piracicaba: Potafós, 1997. 304p.

Mattos Júnior, D.; Quaggio, J. A.; Cantarella, H.; Carvalho, S. A. de. Superfícies de resposta do tangor 'Murcott' à fertilização com N, P e K. Revista Brasileira de Fruticultura, v.26, n.1, p.164-167, 2004.

Melo, L. C. A.; Silva, C. A.; Dias, C. A. Caracterização da matriz orgânica de resíduos de origens diversas. Revista Brasileira de Ciência do Solo, v.32, n.1, p.101-110, 2008.

Melo, W. J.; Marques, M. O.; Melo, V. P. O uso do lodo de esgoto e as propriedades do solo. In: Tsutyia, M. T.; Comparini J. B.; Alem Sobrinho, P.; Hespanhol, I.; Carvalho, P. C. T., Melfi; A. J.; Melo, M. O. (ed.). Lodo de esgotos na Agricultura. São Paulo: SABESP, 2001. p.289-363.

Melo, W. J.; Marques, M. O.; Santiago, G.; Chelli, R. A; Leite, S. A. S. Efeito de doses crescentes de lodo de esgoto sobre frações da matéria orgânica e CTC de um latossolo cultivado com cana-de-açúcar. Revista Brasileira de Ciência do Solo, v.2, n.18, p.449-455, 1994.

Modesto, P. T.; Scabora, M. H.; Colodro, G.; Maltoni, K. L.; Cassiolato, A. M. R. Alterações em algumas propriedades de um latossolo degradado com uso de lodo de esgoto e resíduos orgânicos. Revista Brasileira de Ciência do Solo, v.33, n.5, p.1489-1498, 2009. 
Nogueira, T. A. R.; Oliveira, L. R.; Melo, W. J. de; Fonseca, I. M.; Melo, G. M. P. de; Melo, V. P. de; Marques, M. O. Cádmio, cromo, chumbo e zinco em plantas de milho em latossolo após nove aplicações anuais de lodo de esgoto. Revista Brasileira de Ciência do Solo, v.32, n.5, p.2195-2207, 2008.

Oliveira, F. C.; Mattiazo, M. E.; Marciano, C. R.; Moraes, S. O. Lixiviação de nitrato em um latossolo amarelo distrófico tratado com lodo de esgoto e cultivado com cana-de-açúcar. Scientia Agricola, v.58, n.1, p.171-180, 2001.

Panzenhagen, N. V.; Koller, O. C.; Sartori, I. A.; Portelinha, N. V. Respostas de tangerineiras Montenegrina à calagem e adubação e mineral. Pesquisa Agropecuária Brasileira, v.34, n.4, p.527-533, 1999.

Pedroza, J. P.; Haandel, A. C. van; Beltrão, N. E. de M.; Dionisio, J. A.; Duarte, M. E. M. Qualidade tecnológica da pluma do algodoeiro herbáceo cultivado com biossólidos. Revista Brasileira de Engenharia Agrícola e Ambiental, v.10, n.3, p.586592, 2006

Quaggio, J. A.; Raij, B. van; Piza Júnior, C. T. Faixas de teores adequados de macro e micronutrientes em folhas de plantas frutíferas. In: Raij, B. van; Cantarella, H.; Quaggio, J. A.; Furlani, A. M. C. (ed.). Recomendações de adubação e calagem para o estado de São Paulo. Campinas: Instituto Agronômico, 1997. p.124-125.

Raij, B. van; Andrade, J. C.; Cantarella, H.; Quaggio, J. A. Análise química para avaliação da fertilidade de solos tropicais. Campinas: Instituto Agronômico, 2001. 285p.

Rangel, O. J. P.; Silva, C. A.; Bettiol, W.; Dynia, J. F. Efeito de aplicações de lodos de esgoto sobre os teores de metais pesados em folhas e grãos de milho. Revista Brasileira de Ciência do Solo, v.30, n.3, p.583-594, 2006.
Rocha, G. N.; Gonçalves, J. L. M.; Moura, I. M. Mudanças da fertilidade do solo e crescimento de um povoamento de Eucalyptus grandis fertilizado com lodo de esgoto. Revista Brasileira de Ciência do Solo, v.28, n.4, p.623-639, 2004.

Silva, F. C.; Boaretto, A. E.; Berton, R. S.; Zotelli, H. B.; Pexe, C. A.; Bernardes, E. M. Efeito de lodo de esgoto na fertilidade de um argissolo vermelho-amarelo cultivado com cana-deaçúcar. Pesquisa Agropecuária Brasileira, v.26, n.5, p.831$840,2001$.

Silva, F. C.; Boaretto, A. E.; Berton, R. S.; Zotelli, G. B.; Pexe, C. A.; Mendonça, E. Cana-de-açúcar cultivada em solo adubado com lodo de esgoto. Nutrientes, metais pesados e produtividade. Pesquisa Agropecuária Brasileira, v.13, n.1, p.1-8, 1998.

Silva, J. E.; Resck, D. V. S.; Sharma, R. D. Alternativa agronômica para o lodo de esgoto produzindo no Distrito Federal. II. Aspectos qualitativos, econômicos e práticos do uso. Revista Brasileira Ciência do Solo, v.26, n.2, p.497-503, 2002.

Simonete, M. A.; Kiehl, J. C. Extração e fitodisponibilidade de metais pesados em resposta à adição de lodo de esgoto. Scientia Agricola, v.59, n.3, p.555-563, 2002.

Simonete, M. A.; Kiehl, J. C.; Andrade, C. A.; Teixeira, C. F. A. Efeito do lodo de esgoto em um argissolo e no crescimento e nutrição de milho. Pesquisa Agropecuária Brasileira, v.38, n.10, p.1187-1195, 2003.

Trannin, I. C. de; Siqueira, J. O.; Moreira, F. M. S. Atributos químicos e físicos de um solo tratado com lodo de esgoto industrial e cultivado com milho. Revista Brasileira de Engenharia Agrícola e Ambiental, v.12, n.3, p.223-230, 2008.

Tubelis, A; Salibe, A. A. Relação entre a produção de laranjeira 'Hamlin' e as precipitações mensais no altiplano Botucatu. Pesquisa Agropecuária Brasileira, v.24, p.801-806, 1989. 